\title{
Clinicopathological Study of Nephrotic Syndrome in Indian Children: A Tertiary Care Experience
}

\author{
Chetan S. Chaudhari*, Nitin M.Gadgil, Prashant Vijay Kumavat, Ganesh Ramdas Kshirsagar, Sagar A Dhamne \\ Dept of Pathology, LTMMC \& LTMGH, Sion, Mumbai, India
}

\section{ABSTRACT}

Background: Nephrotic syndrome is an important chronic disorder in children with clinical manifestation of different histopathological subtypes. An aim of the study was to determine incidence of renal biopsy in paediatric nephrotic syndrome, correlations between the clinical and histomorphological patterns in Indian ethnicity children at our tertiary care institute.

Methods: A retrospective study of kidney biopsies with, immunofluorescence and electron microscopy with clinical outcome of the children with nephrotic syndrome was done over a period of 15 years. Biopsies were stained with Hematoxylin \& eosin, Periodic acid Schiff and silver impregnation stain and also processed for immunofluorescence and electron microscopy as necessary. All renal biopsy findings were correlated with clinical response to steroid therapy, immunosuppressant, and clinical parameters.

Result: Frequencies in results were calculated by Chi square test for categorical variables. Mann-Whitney U test was used for non parametric variables. Predominantly cases were seen in the age group 8-12 years. Atypical nephrotic syndrome was the commonest presenting feature followed by steroid resistant nephrotic syndrome. Minimal change disease was the commonest histomorphological pattern followed by focal segmental glomerular sclerosis. All the cases of membranous glomerulonephritis in study were secondary in nature. Light microscopy findings correlated with electron microscopy, in $80.95 \%$ cases.

Conclusion: Minimal change disease occurs up to 6 times more commonly in Indian children than in Europian counterpart, but rise in FSGS cases as a cause for nephrotic syndrome is now being encountered. In India the proportion of cases with Membrano-proliferative glomerulonephritis is high, attributable to the high prevalence of infectious diseases like tuberculosis, chronic suppurative infections and malnutrition

\section{Keywords: Paediatric, Nephrotic Syndrome, Electron Microscopy, Immunofluoresence}

\section{Introduction}

Nephrotic syndrome is an important chronic disorder in children, characterized by heavy proteinuria, hypoalbuminemia, hyperlipidemia and edema. About $90 \%$ children with idiopathic nephrotic syndrome have 'minimal lesion' on renal histological examination and respond promptly to corticosteroid therapy with remission of proteinuria. Approximately three-fourths of these patients have one or more relapses that require repeated treatment with corticosteroids. A small proportion of patients who are steroid resistant are at risk of complications and renal insufficiency. Estimates on the annual incidence of nephrotic syndrome range from 2-7 per 100,000 children, and prevalence from $12-16$ per 100,000 . There is epidemiological evidence of a higher incidence of nephrotic syndrome in children from south Asia. A prospective double blind study of nephrotic syndrome was performed for the International Study of Kidney Disease in Children demonstrated minimal change nephrotic syndrome was the commonest histological lesion and focal segmental glomerular sclerosis (FSGS) as a rare cause in children. ${ }^{[1]}$ However, recent studies like by Bonilla-Felix et al and by Gulati et al ${ }^{[2]}$ in India have found rise in FSGS cases as a cause for nephrotic syndrome with specific ethnic relationship. In the present retrospective study of over last 15 years, our aims and objectives were to study incidence of renal biopsy in paediatric nephrotic syndrome, correlations between the clinical and histomorphological patterns with clinical outcome in Indian ethnicity children at our tertiary care institute. Renal biopsy findings were also correlated on light microscopy, immunofluorescence and on electron microscopy as necessary.

\section{Materials and Methods}

A retrospective study of kidney biopsies from the children with nephrotic syndrome was done over a period of 15 years at a tertiary care centre with a capacity of 1700 beds. Total 92 renal biopsies were received. At the time of biopsy two cores were obtained whenever possible. One core was fixed in $10 \%$ formal saline and embedded in paraffin,4-5 $\mu \mathrm{m}$ thick sections were cut and were stained with Hematoxylin \& eosin, Periodic acid Schiff (PAS) and silver impregnation stain. The ends of other core were cut and fixed in $3 \%$ gluteraldehyde for 
electron microscopic examination and the remaining core was wrapped in a foil on saline soaked gauze and sent in ice for immunofluorescence staining using antibodies for IgG, IgA, IgM and C3. On light microscopy the lesions were scrutinised first for adequacy. At least 5 glomeruli were considered for the biopsy to be adequate; however the changes, if diagnostic and pathognomic of the lesion were seen in even one glomerulus then, the biopsy was termed adequate. Depending on histomorphology cases were diagnosed as Minimal change disease, FSGS, Mesangial proliferative glomeulonephritis, IgM nephropathy, Membranous glomeulonephritis, Membranoproliferative glomeulonephritis, IgA nephropathy and others. On immunofluorescence study lesions were interpreted for presence or absence of antibodies for $\operatorname{IgG}, \operatorname{IgM}, \operatorname{Ig} \mathrm{A}, \mathrm{C} 3$, pattern of positivity whether lumpy, granular or linear and site of positivity whether mesangial or capillary basement. The electron microscopy findings were correlated in specific cases. All renal biopsy findings were studied and correlated with clinical response to steroid therapy, immunosuppressant, and other relevant clinical parameters. The response to the therapy was defined as per the guidelines set by the consensus statement of Indian Paediatric Nephrology group, Indian Academy of Paediatrics.

\section{Result}

A total of 433 biopsies were received over the study period.261 (60.28\%) biopsies were from patients with nephrotic syndrome. Of the 261 cases of nephrotic syndrome 84 biopsies $(32.18 \%)$ were from the pediatric age group (up to 12 years of age). Out of the 84 biopsies performed 70 were adequate as per criteria for adequacy. Predominantly cases were seen in the age group 8-12 years $(50 \%)$, followed by $4-8$ years $(31.42 \%)$. An overall male predominance was noted with Male: Female ratio 1.59:1(Fig: 1). Steroid resistant nephrotic syndrome ( SrNS $: 34.28 \%$ ) was the commonest presenting feature in children undergoing renal biopsy followed by Atypical nephrotic syndrome (27.14\%) and frequently relapsing nephrotic syndrome ( FRNS: 24.28\%).(Table 1) Minimal change disease (MCD) (33 cases 47.14\%) was the commonest histomorphologic pattern in pediatric nephrotic syndrome followed by focal segmental glomerular sclerosis (FSGS)(16 cases, 22.85\%).Mesangial proliferative glomerulonephritis (MesPGN) was seen in 5 cases (7.14\%), while 4 cases $(5.71 \%)$ each of IgM nephropathy and membranoproliferative glomerulonephritis (MPGN), 3 cases $(4.28 \%)$ each of membranous and IgA nephropathy were seen. One case of resolving acute post streptococcal glomerulonephritis presented as nephrotic syndrome. One case of congenital nephrotic syndrome and 1 case of familial nephrotic syndrome were also noted.
Among the SrNS cases the commonest histomorphologic pattern was FSGS (41.66\%), followed by MCD (33.53\%). Out of 17 cases presenting as FRNS, MCD was (76.47\%) the commonest histomorphologic pattern. Among the 19 cases of atypical nephrotic syndrome, MCD was the commonest cause ( 8 cases, $42.10 \%$ ) followed by $\operatorname{IgM}$ and $\operatorname{IgA}$ nephropathy (15.78\%) each. We had 7 cases of steroid dependant nephrotic syndrome. Commonest histomorphologic patterns noted were MCD and FSGS (3 cases each) (Table 2).

MCD was commonly seen in children more than 8 years of age (17 cases). An overall male predominance was noted with sex ratio of 2.18:1 (Table 3). Of the 33 cases of MCD, follow up was available in 16 cases. Eight cases (50\%) attained remission after steroid therapy alone. Four cases $(25 \%)$ responded to immunosuppressants while 3 failed to respond to either treatment modality. Four Cases presenting with atypical nephrotic syndrome responded to steroid therapy alone. One case of familial nephrotic syndrome was noted, which presented as FRNS and succumbed to death. Immunofluorescence was available in 9 cases. Only 2 cases showed non specific weak positivity for $\operatorname{IgM}$ and IgG. MesPGN was commonly seen in children less than 4 years of age. Of the 5 cases of MesPGN, 3 cases did not respond to steroid or immunosuppressive therapy. One case of SRNS responded to immunosuppressant therapy. One case which presented in renal failure died. In 1 case capillary IgM deposits on immunofluorescence was seen. Of 4 cases of $\operatorname{IgM}$ nephropathy follow up was available in 3 cases. 1 case responded to steroid therapy alone, 1 case failed to respond to steroid as well as immunosuppressant therapy, while 1 case died .All the cases showed mesangial IgM deposits on immunofluorescence and it was the only and the brightest deposit seen. (Table 4) FSGS was equally seen in the age groups 4-8 years and 8-12 years with sex ratio of $1.8: 1$. Peripheral location was the commonest location for sclerosis within the glomeruli in focal segmental glomerular sclerosis, seen in 7 out of 16 cases of FSGS. Two cases each of collapsing glomerulopathy and perihilar sclerosis were noted. 1 case of glomerular tip lesion was also noted. Interstitial changes were noted in 10 cases of which 8 cases showed interstitial fibrosis. 2 cases revealed tubular atrophy. Of the 2 cases where immunofluorescence was available 1 case showed IgM deposits in the mesangium..(Table 5)

All the cases of membranous glomerulonephritis (MGN) in our study were secondary in nature. Two cases were positive for Hepatitis B surface antigen (HBsAg) presented as steroid resistant nephrotic syndrome and as atypical nephrotic syndrome each and one case was positive for 
Antinuclear antibody (ANA), presented as steroid resistant nephrotic syndrome. Both the cases presenting as steroid resistant nephrotic syndrome showed IgG deposits in the mesangium. The case with ANA positivity showed immune deposits of IgG, IgM and C3.Two cases each of primary and secondary MPGN were noted. Both the cases of idiopathic MPGN were steroid resistant, while both the cases of secondary form presented with hematuria as atypical nephrotic syndrome. Of the 2 cases of secondary MPGN one was positive for antinuclear antibody (ANA) and the other had a positive Mantoux test and diagnosed as pulmonary tuberculosis. (Table 6) All the 3 cases of IgA nephropathy presented with hematuria along with nephrotic syndrome as atypical nephrotic syndrome. The glomeruli revealed increase in mesangial cellularity in all the cases. IgA positivity in the mesangium was the predominant finding on immunofluorescence. Two cases also showed IgM positivity while $\operatorname{IgG}$ positivity was seen in one case. Electron microscopy was available in 36 cases. 5 biopsies $(13.88 \%)$ were inconclusive while 7 (19.44\%) biopsies were inadequate.16 biopsies (44.44\%) were consistent with the diagnosis of minimal change. (Table 7) Light microscopy findings correlated with electron microscopy findings, in 20 cases out of the 24 cases where electron microscopic diagnosis was available. Discrepancy was seen in 4 cases. Out of these, 3 cases which were diagnosed as FSGS on light microscopy, 2 cases were diagnosed as minimal change and 1 case was diagnosed as diffuse mesangial proliferation on electron microscopy. One case diagnosed as IgA nephropathy on light microscopy and immunofluorescence showed only foot process effacement without mesangial deposits on electron microscopy. (Table 7)

Table 1: Age wise distribution of nephrotic syndrome in children as per clinical presentation.

\begin{tabular}{|l|c|c|c|c|}
\hline & $\mathbf{0 - 4}$ yrs & $\mathbf{4 - 8}$ yrs & $\mathbf{8 - 1 2}$ yrs & Total \\
\hline SrNS & 05 & 08 & 11 & $24(34.28 \%)$ \\
\hline FRNS & 03 & 08 & 06 & $17(24.28 \%)$ \\
\hline Atypical & 02 & 05 & 12 & $19(27.14 \%)$ \\
\hline SDNS & 01 & 01 & 05 & $07(10.00 \%)$ \\
\hline Renal Failure & 01 & -- & -- & $01(1.42 \%)$ \\
\hline New cases & 01 & -- & 01 & $02(2.85 \%)$ \\
\hline Total & $\mathbf{1 3}$ & $\mathbf{2 2}$ & $\mathbf{3 5}$ & $\mathbf{7 0}$ \\
\hline
\end{tabular}

(SrNS- steroid resistant nephrotic syndrome, FRNS- frequently relapsing nephrotic syndrome, SDNS- steroid dependant nephrotic syndrome)

Table 2: Distribution of cases as per clinical presentation and the histomorphologic pattern in pediatric nephrotic syndrome.

\begin{tabular}{|l|c|c|c|c|c|c|c|}
\hline CP HP & SrNS & FRNS & Atypical & SDNS & Renal failure & New cases & Total \\
\hline MCD & $8(33.33)$ & $13(76.47)$ & $8(42.10)$ & $3(42.85)$ & -- & $1(50)$ & $33(47.14)$ \\
\hline FSGS & $10(41.66)$ & $2(11.76)$ & $1(5.26)$ & $3(42.85)$ & -- & -- & $16(22.85)$ \\
\hline MesPGN & $2(8.33)$ & $1(5.88)$ & -- & $1(14.28)$ & $1(100)$ & -- & $5(7.14)$ \\
\hline IgM & -- & $1(5.88)$ & $3(15.78)$ & -- & -- & -- & $4(5.71)$ \\
\hline MGN & $2(8.33)$ & -- & $1(5.26)$ & -- & -- & -- & $3(4.28)$ \\
\hline MPGN & $2(8.33)$ & -- & $2(10.52)$ & -- & -- & -- & $4(5.71)$ \\
\hline IgA & -- & -- & $3(15.78)$ & -- & -- & -- & $3(4.28)$ \\
\hline APSGN & -- & -- & $1(5.26)$ & -- & -- & -- & $1(1.42)$ \\
\hline Cong. NS & -- & -- & -- & -- & -- & $1(50)$ & $1(1.42)$ \\
\hline Total & $\mathbf{2 4}$ & $\mathbf{1 7}$ & $\mathbf{1 9}$ & $\mathbf{7}$ & $\mathbf{1}$ & $\mathbf{2}$ & $\mathbf{7 0}$ \\
\hline
\end{tabular}

(HP- histomorphologic pattern, CP-clinical presentation, MCD- minimal change disease, FSGS- focal segmental glomerulosclerosis, MesPGN- mesangial proliferative glomerulonephritis, IgM- IgM nephropathy, MGN- membranous glomerulonephritis, MPGN- membranoproliferative glomerulonephritis, IgAIgA nephropathy, APSGN- acute post streptococcal glomerulonephritis, Cong. NS- congenital nephrotic syndrome N.B. Figures in parenthesis indicate percentage.) 
Table 3: Age wise distribution of various histomorphologic patterns of paediatric nephrotic syndrome

\begin{tabular}{|c|c|c|c|c|c|c|c|}
\hline Age/HP & $0-4$ yrs & $4-8$ yrs & 8-12 yrs & Total & Males & Females & Ratio \\
\hline MCD & 7 & 9 & 17 & 33 & 24 & 11 & 2.18:1 \\
\hline FSGS & 2 & 7 & 7 & 16 & 9 & 5 & 1.8:1 \\
\hline MesPGN & 3 & 1 & 1 & 5 & 3 & 2 & $1.5: 1$ \\
\hline $\lg M$ & -- & 1 & 3 & 4 & 3 & 1 & $3: 1$ \\
\hline MGN & -- & 2 & 1 & 3 & 2 & 1 & $2: 1$ \\
\hline MPGN & -- & -- & 4 & 4 & -- & 4 & -- \\
\hline $\lg A$ & -- & 2 & 1 & 3 & 1 & 2 & $1: 2$ \\
\hline APSGN & -- & -- & 1 & 1 & -- & 1 & -- \\
\hline Cong NS & 1 & -- & -- & 1 & 1 & -- & -- \\
\hline Total & 13 & 22 & 35 & 70 & 43 & 27 & 1.59:1 \\
\hline
\end{tabular}

(HP: Histomorphologic pattern)

Table 4: Correlation between clinical presentation, clinical outcome and immunofluorescence findings in MCD , MesPGN \& IgM nephropathy .

\begin{tabular}{|c|c|c|c|c|c|c|c|c|c|c|c|c|c|c|}
\hline \multirow{9}{*}{ MCD } & \multirow[b]{3}{*}{$\begin{array}{c}\text { Clinical } \\
\text { presentation }\end{array}$} & \multirow[b]{3}{*}{$\begin{array}{l}\text { No. of } \\
\text { Cases }\end{array}$} & \multicolumn{4}{|c|}{ Clinical Outcome } & \multicolumn{8}{|c|}{ Immunofluorescence } \\
\hline & & & \multirow[b]{2}{*}{$\begin{array}{c}\text { Remission } \\
\text { after } \\
\text { steroid } \\
\text { therapy }\end{array}$} & \multirow[b]{2}{*}{$\begin{array}{l}\text { Remission } \\
\text { after Immuno } \\
\text { suppressants }\end{array}$} & \multirow[b]{2}{*}{$\begin{array}{c}\text { Non } \\
\text { responders }\end{array}$} & \multirow[b]{2}{*}{ Deaths } & \multicolumn{2}{|c|}{$\lg G$} & \multicolumn{2}{|c|}{$\lg M$} & \multicolumn{2}{|c|}{$\lg A$} & \multicolumn{2}{|c|}{ C3 } \\
\hline & & & & & & & 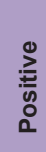 & 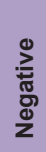 & $\sum_{\substack{0 \\
0}}^{0}$ & 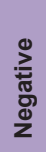 & 竞 & 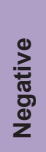 & 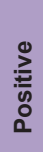 & 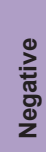 \\
\hline & SrNS & 08 & -- & 02 & -- & -- & 01 & 02 & 01 & 02 & -- & 03 & -- & 03 \\
\hline & FRNS & 13 & 03 & 01 & 03 & 01 & 01 & 03 & 01 & 03 & -- & 04 & -- & 04 \\
\hline & SDNS & 03 & -- & 01 & -- & -- & -- & 01 & -- & 01 & -- & 01 & -- & 01 \\
\hline & Atypical & 08 & 04 & -- & -- & -- & -- & 01 & -- & 01 & -- & 01 & -- & 01 \\
\hline & New cases & 01 & 01 & -- & -- & -- & -- & -- & -- & -- & -- & -- & -- & -- \\
\hline & Total & 33 & 08 & 04 & 03 & 01 & 02 & 07 & 02 & 07 & -- & 09 & -- & 09 \\
\hline \multirow{6}{*}{ MesPGN } & SrNS & 02 & -- & 01 & 01 & -- & -- & 01 & -- & 01 & -- & 01 & -- & 01 \\
\hline & FRNS & 01 & -- & -- & 01 & -- & -- & 01 & 01 & -- & -- & 01 & 01 & -- \\
\hline & SDNS & 01 & -- & -- & 01 & -- & -- & -- & -- & -- & -- & -- & -- & -- \\
\hline & Atypical & -- & -- & -- & -- & -- & -- & -- & -- & -- & -- & -- & -- & -- \\
\hline & New cases & 01 & -- & -- & -- & 01 & -- & -- & -- & -- & -- & -- & -- & -- \\
\hline & Total & 05 & -- & 01 & 03 & 01 & -- & 02 & 01 & 01 & -- & 02 & 01 & 01 \\
\hline \multirow{5}{*}{$\begin{array}{l}\text { IgM } \\
\text { Nephropathy }\end{array}$} & SrNS & -- & -- & -- & -- & -- & -- & -- & -- & -- & -- & -- & -- & -- \\
\hline & FRNS & 01 & 01 & -- & -- & -- & -- & 01 & 01 & -- & -- & 01 & -- & 01 \\
\hline & SDNS & -- & -- & -- & -- & -- & -- & -- & -- & -- & -- & -- & -- & -- \\
\hline & Atypical & 03 & -- & -- & 01 & 01 & 01 & 02 & 03 & -- & -- & 03 & 01 & 02 \\
\hline & Total & 04 & 01 & -- & 01 & 01 & 01 & 03 & 04 & -- & -- & 04 & 01 & 03 \\
\hline
\end{tabular}


Table 5: Correlation between the various histologic variants of focal segmental glomerulonephritis,clinical presentation and immunofluorescence findings

\begin{tabular}{|c|c|c|c|c|c|c|c|c|c|c|c|c|c|c|c|c|c|c|c|c|c|}
\hline \multirow{9}{*}{ FSGS } & & \multicolumn{8}{|c|}{ Histomorphology } & \multicolumn{8}{|c|}{ Immunofluorescence } & \multirow{3}{*}{ 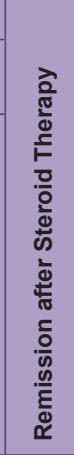 } & \multirow{3}{*}{ 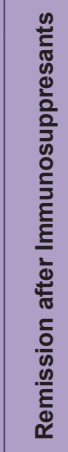 } & \multirow[b]{3}{*}{$\begin{array}{l}\frac{n}{0} \\
\frac{0}{0} \\
\frac{0}{0} \\
\frac{0}{0} \\
0 \\
\frac{0}{\alpha} \\
\text { ㅇ }\end{array}$} & \multirow[b]{3}{*}{$\begin{array}{l}\text { 뎽 } \\
\text { ه }\end{array}$} \\
\hline & & \multicolumn{5}{|c|}{ Glomerular changes } & \multicolumn{2}{|c|}{$\begin{array}{l}\text { Tubulo- } \\
\text { interstitium }\end{array}$} & \multirow[b]{2}{*}{ 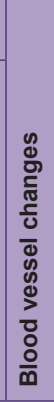 } & \multicolumn{2}{|l|}{$\lg G$} & \multicolumn{2}{|c|}{$\lg M$} & \multicolumn{2}{|c|}{$\lg A$} & \multicolumn{2}{|l|}{ C3 } & & & & \\
\hline & 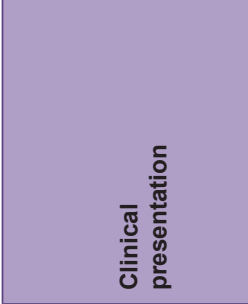 & 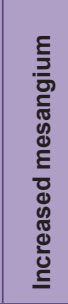 & 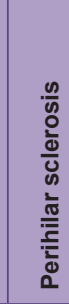 & $\frac{\frac{5}{y}}{\frac{0}{0}}$ & 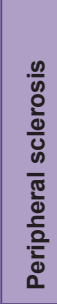 & 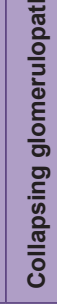 & 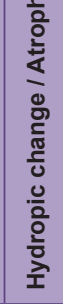 & $\begin{array}{l}\frac{0}{0} \\
\frac{0}{0} \\
\frac{0}{2}\end{array}$ & & 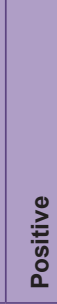 & 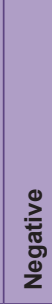 & 道 & 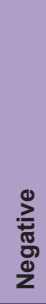 & 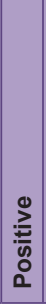 & 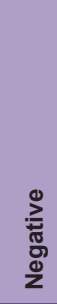 & 道 & 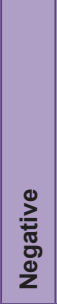 & & & & \\
\hline & Sr NS (10 cases) & 03 & 01 & 01 & 04 & 01 & -- & 05 & -- & -- & -- & -- & -- & -- & -- & -- & -- & -- & 01 & 02- & -- \\
\hline & FRNS (02 cases) & 01 & -- & --- & 01 & 01 & 01 & 01 & -- & -- & 01 & -- & 01 & -- & 01 & - & 01 & -- & -- & -- & -- \\
\hline & SDNS (03 cases) & 02 & 01 & -- & 01 & -- & 01 & 01 & -- & -- & 01 & 01 & -- & 01 & -- & -- & 01 & -- & -- & -- & -- \\
\hline & Atypical (01 case) & -- & -- & -- & 01 & -- & -- & 01 & -- & -- & -- & -- & -- & -- & -- & -- & -- & -- & -- & -- & -- \\
\hline & Renal Failure (None) & -- & -- & -- & -- & -- & -- & -- & -- & -- & -- & -- & -- & -- & -- & -- & -- & -- & -- & -- & -- \\
\hline & Total (16 cases) & 06 & 02 & 01 & 07 & 02 & 02 & 08 & -- & -- & 02 & 01 & 01 & 01 & 01 & -- & 02 & -- & 02 & 02 & -- \\
\hline
\end{tabular}

Table 6: Clinical presentation and immunofluorescence findings in MGN \& MPGN in pediatric nephrotic syndrome

\begin{tabular}{|c|c|c|c|c|c|c|c|c|c|c|c|c|c|}
\hline \multirow{8}{*}{ MGN } & \multirow[b]{3}{*}{$\begin{array}{c}\text { Clinical } \\
\text { presentation }\end{array}$} & \multirow[b]{3}{*}{$\begin{array}{l}\text { No of } \\
\text { case }\end{array}$} & \multirow[b]{3}{*}{$\begin{array}{c}\text { Idio } \\
\text { pathic }\end{array}$} & \multirow{2}{*}{\multicolumn{2}{|c|}{ Secondary }} & \multicolumn{8}{|c|}{ Imnunofuorescence } \\
\hline & & & & & & \multicolumn{2}{|c|}{$\lg G$} & \multicolumn{2}{|c|}{$\operatorname{Ig} A \mid$} & \multicolumn{2}{|c|}{$\lg A$} & \multicolumn{2}{|c|}{ C3 } \\
\hline & & & & 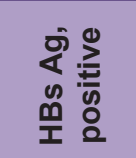 & 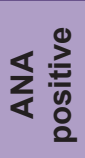 & 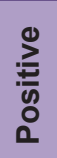 & 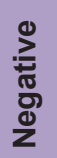 & 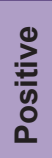 & 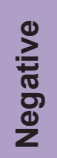 & 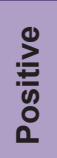 & 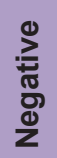 & 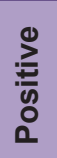 & 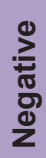 \\
\hline & SrNS & 02 & -- & 01 & 01 & 02 & -- & 01 & 01 & -- & 02 & 01 & 01 \\
\hline & FRNS & -- & -- & -- & -- & -- & -- & -- & -- & -- & -- & -- & -- \\
\hline & SDNS & -- & -- & -- & -- & -- & -- & -- & -- & -- & -- & -- & -- \\
\hline & Atypical & 01 & -- & 01 & -- & -- & -- & -- & -- & -- & -- & -- & -- \\
\hline & Total & 03 & -- & 02 & 01 & 02 & -- & 01 & 01 & -- & 02 & 01 & 01 \\
\hline \multirow{8}{*}{ MPGN } & \multirow[b]{3}{*}{$\begin{array}{c}\text { Clinical } \\
\text { presentation }\end{array}$} & \multirow[b]{3}{*}{$\begin{array}{l}\text { No. } \\
\text { of } \\
\text { case }\end{array}$} & \multirow[b]{3}{*}{$\begin{array}{c}\text { Idio } \\
\text { pathic }\end{array}$} & \multicolumn{2}{|c|}{ Secondary } & \multicolumn{8}{|c|}{ Immunofluorseccene } \\
\hline & & & & \multirow[b]{2}{*}{$\begin{array}{c}\text { MT } \\
\text { positive }\end{array}$} & \multirow[b]{2}{*}{ 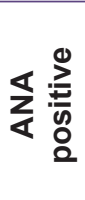 } & \multicolumn{2}{|c|}{$\lg G$} & \multicolumn{2}{|c|}{$\lg M$} & \multicolumn{2}{|c|}{$\lg A$} & \multicolumn{2}{|c|}{ C3 } \\
\hline & & & & & & 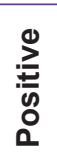 & 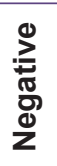 & 竞 & 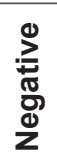 & 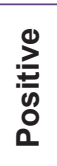 & 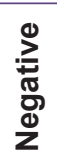 & 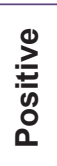 & 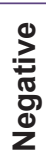 \\
\hline & STNS & 02 & 02 & -- & -- & 01 & -- & -- & 01 & 01 & -- & 01 & -- \\
\hline & FRNS & -- & -- & -- & -- & -- & -- & -- & -- & -- & -- & -- & -- \\
\hline & SDNS & -- & -- & -- & -- & -- & -- & -- & -- & -- & -- & -- & -- \\
\hline & Atypical & 02 & -- & 01 & 01 & 01 & -- & -- & 01 & -- & 01 & 01 & -- \\
\hline & Total & 04 & 02 & 01 & 01 & 02 & -- & -- & 02 & 01 & 01 & 02 & -- \\
\hline
\end{tabular}

(HBsAg-Hepatitis B surface antigen,ANA-Antinuclear antibody, MT-Mantoux test)

Table 7: Frequency of histomorphologic patterns on electron microscopy.

\begin{tabular}{|l|c|c|}
\hline Histomorphologic pattern & No. of cases & Percentage \\
\hline MCD & 16 & 44.44 \\
\hline FSGS & 03 & 8.33 \\
\hline MesPGN & 02 & 5.55 \\
\hline
\end{tabular}




\begin{tabular}{|l|c|c|}
\hline Histomorphologic pattern & No. of cases & Percentage \\
\hline MGN & 01 & 2.77 \\
\hline MPGN & 01 & 2.77 \\
\hline APSGN & 01 & 2.77 \\
\hline Inconclusive & 05 & 13.88 \\
\hline Inadequate & 07 & 19.44 \\
\hline Total & 36 & 100 \\
\hline
\end{tabular}

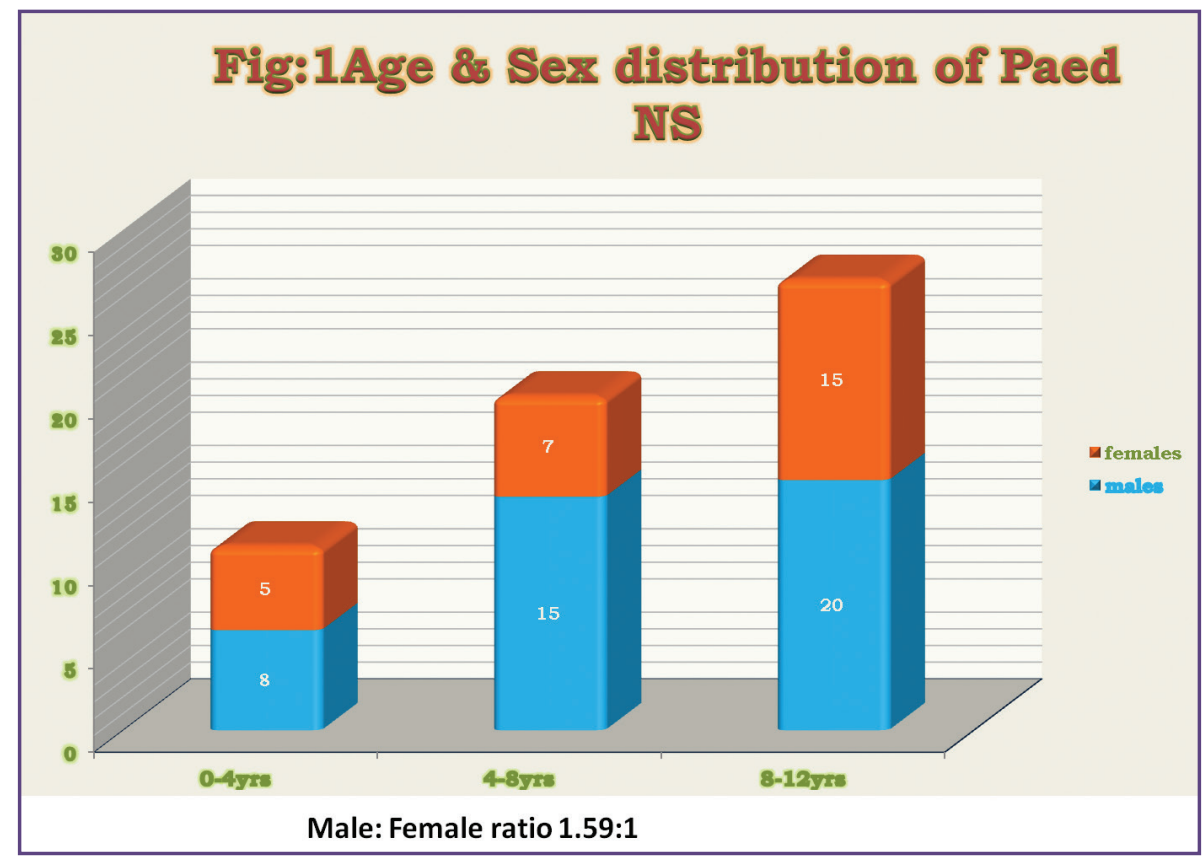

Fig. 1: Age \& sex distribution of Paediatric Nephrotic Syndrome cases.

\section{Discussion}

Nephrotic syndrome in children is a clinical manifestation of different histopathological subtypes. Minimal change disease remains the most common cause of idiopathic nephrotic syndrome, especially in children under 8 years of age. The distribution of glomerular lesions in children differs from that in adults. The country of origin may affect the proportions of the diseases causing the nephrotic syndrome. MCD occurs up to 6 times more commonly in some Asian (India, Pakistan, Bangladesh) children than in Caucasian counterparts in Europe. ${ }^{[3]}$ In the Asian countries the proportion of cases with MPGN is high, attributable to the high prevalence of infectious diseases like leprosy, tuberculosis, chronic suppurative infections and malnutrition.Most of the children with nephrotic syndrome not having hematuria, hypertension, or impaired renal function are treated initially without undergoing a kidney biopsy. A biopsy is usually not indicated in patients with frequent relapses when treatment with Levamisole or Cyclophosphamide is being considered. A biopsy should be performed before starting Cyclosporine therapy and indicated in children $<1$ year or $>15$ years, patients with persistent microscopic/gross hematuria, low C3,sustained hypertension, renal failure, suspecting secondary causes of nephrotic syndrome, frequently relapsing,steroid dependant nephrotic syndrome. ${ }^{[4]}$

Out of the 24 cases of steroid resistant nephrotic syndrome, 8 cases were in the age group 4-8 years and 11 cases were in the 8-12 years age group, with a slight male predominance [Table 1]. Gulati et al. observed that FSGS was the commonest histologic type $(58.8 \%)$ followed by MCD (17.6\%), MesPGN (17.6\%), MGN and MPGN (4\%) each as seen in our study. Minimal change disease and nonminimal change diseases almost equally attained remission ( 2 cases versus 3 cases respectively).In the study done by Gulati et al. MCD had greater remission rates as compared to non-minimal change disease $(\mathrm{P}=<0.00001)$.

We had 33 cases $(47.14 \%)$ of minimal change disease with male predominance. Histology revealed unremarkable 
glomeruli on hematoxylin \& eosin, PAS and silver staining (fig 2A,B,C). Immunofluorescence was possible in 9 cases, out of which 2 cases showed non specific positivity for $\operatorname{IgM}$ and $\operatorname{IgG}$. Electron microscopy revealed flattening and fusion of the foot processes of the visceral epithelial cells (fig 2D).In the study by Jitendra Kumar et al. ${ }^{\left[{ }^{[5]}\right.} \mathrm{MCD}$ was seen in 95 of the 290 cases (32.76\%). Majority of the cases were seen in the age greater the 8 years with a male predominance (3:1).Atypical nephrotic syndrome was seen in 18 of the 95 cases (18.95\%) of MCD. Atypical nephrotic syndrome was seen as clinical presentation in $36 \%$ of the cases in study by Habib $\mathrm{R}^{[6]}$ and in $13 \%$ of the cases in a study by White RH et al. ${ }^{[7]}$ In study by Tejani A, frequently relapses were seen in $40 \%$ of the cases, while $15 \%$ cases relapsed infrequently. Recent studies have suggested that IL-4 and IL-13 are overexpressed in patients with steroidresponsive nephrotic syndrome in relapse. It is of interest that these same cytokines can increase transcellular ion transport over glomerular visceral epithelial cell monolayers ${ }^{[8]}$. These effects on ion transport are associated with basolateral secretion of lysosomal proteinases, such as procathepsin L. Such effects may link abnormal cytokine expression to altered glomerular permeability. Some of the data, such as up-regulation of c-maf expression and downregulation of IL-12Rß2 during relapse could fit well into previously proposed paradigms of "Th2-bias" in patients with active $\mathrm{MCNS}^{[9]}$.In addition, levels of IL4 and CD 23 (a receptor for IgE ) have been found to be elevated in the peripheral lymphocytes. Synaptopodin is a proline-rich protein intimately associated with actin microfilaments present in the foot processes of podocytes. Greater synaptopodin expression in the podocytes is associated with a significantly better response to steroid therapy ${ }^{[10]}$

We had 16 cases $(22.85 \%)$ of FSGS with a significant male predominance (M:F ratio 1.8:1). SRNS was the commonest clinical presentation followed by steroid dependant nephrotic syndrome and frequently relapsing syndrome. Histology revealed 7 cases with peripheral sclerosis, 2 cases each with collapsing glomerulopathy and perihilar sclerosis and 1 case with tip lesion (fig.3 A,B,C,D). Increased mesangial cellularity was noted in 6 cases $(50 \%)$. One case out of the 2 cases where immunofluorescence was available, showed IgM positivity in the mesangium. Electron microscopy showed focal areas of sclerosis (fig 3E). In one case wrinkling of the glomerular basement membrane was also noted (fig 3F). Southwest Pediatric Nephrology Study Group found that $48 \%$ had focal mesangial hypercellularity and 39\% had diffuse mesangial cellularity amongst FSGS. ${ }^{[1]}$ FSGS has been found to be more prevalent in the study carried out at SGPGI Lucknow in the Indian children and was the most common histological subtype in children in steroid resistant nephrotic syndrome. Also a study in Houston suggested an increasing incidence of FSGS among children which was race independent. The study concluded that the incidence of FSGS in children with nephrotic syndrome has increased significantly in the recent years. In addition, FSGS is the most common cause of nephrotic syndrome in African-Americans ${ }^{[12]}$

We had 5 cases $(8.62 \%)$ of MesPGN. 2 cases $(40 \%)$ presented clinically with steroid resistant nephrotic syndrome while there was 1 case $(20 \%)$ each of frequently relapsing and steroid dependant nephrotic syndrome. Histology revealed increased mesangial cellularity on haematoxylin and eosin (fig 4A,B). Only one case revealed IgM and $\mathrm{C} 3$ deposits. Thus a poor outcome was noted with mesangial proliferative nephrotic syndrome. We have separated MesPGN from MCD as the outcome of these cases is different from minimal change disease as also observed by Waldherr and Habib, who in their study found that in 6 patients $(33.33 \%)$ out of 18 had renal failure and in $5(27.78 \%)$ remission was seen. ${ }^{[13]}$ Remaining patients continued to have nephrotic syndrome. Bernstein and Edelmann suggested that presence of diffuse mesangial hypercellularity may indicate more severe injury that could require additional therapy to induce good response. ${ }^{[14]}$

We had 4 cases $(6.90 \%)$ of IgM nephropathy. 3 out of the 4 cases had atypical nephrotic syndrome i.e. hematuria with nephrotic syndrome On histology the glomeruli were unremarkable except in one case where mild increase in mesangial cellularity was noted. On immunofluorescence IgM was seen predominantly seen in the mesangium and it was the only and the brightest deposit (fig 4C,D).In the study by Hirszel et al. 7 out 9 cases $(77.78 \%)$, belonged to either steroid resistant or steroid dependant, frequently relapsing group and only 1 (14.29\%) of them developed progressive renal failure. ${ }^{[15]}$

We had 3 cases (5.17\%) of membranous glomerulonephritis (MGN). All the 3 cases were more than 4 years in age. 2 were males and 1 was a female. Histology revealed thickening of the capillary basement membranes confirmed on periodic acid Schiff (PAS) staining.The silver stain showed spikes along the capillary basement membrane.On immunofluorescence granular IgG deposits were typically seen along the glomerular capillary wall (fig 5A,B,C). Electron microscopy revealed subepithelial deposits in contact with and indenting the visceral epithelial cells (fig5D). Gulati et al. in their study had 6 cases $(4.4 \%)$ of MGN, with a male predominance (5 males, 1 female). 2 cases presented as atypical nephrotic syndrome while other 4 cases were steroid resistant. IgA nephropathy was seen in 3 cases $(5.17 \%)$. All the cases presented as atypical nephrotic syndromes. Histology revealed a mild 
to moderate increase in mesangial cellularity while the rest of the glomerulus was unremarkable. Immunofluorescence showed predominant diffuse global mesangial IgA deposits (Fig 5E). One case, in addition was positive for IgM and IgG. Electron microscopy was available in none of the cases.In IgA nephropathy macroscopic hematuria is seen $30 \%-35 \%$ patients although it may occur subsequently in another $10-15 \%$. Equal number of patients (30-35\%) present for investigation of asymptomatic proteinuria, that is usually accompanied by microscopic hematuria. Berger $\mathrm{J}$ and Hinglias $\mathrm{N}$ observed that granular deposits of IgA and $\mathrm{C} 3$ are present predominantly in the mesangium but there may be variable deposits of $\mathrm{IgG}$, IgM or both, frequently fluorescing with lesser intensity. ${ }^{[16]}$ We had one case which presented as atypical nephrotic syndrome. Biopsy revealed features of proliferative glomerulonephritis secondary to streptococcal infection, which was in the resolving phase. In their study Sagel et al. found that subclinical disease was almost 20 times more common than overt acute glomerulonephritis. ${ }^{[17]}$ Out of 54 children, 15 children $(27.78 \%)$ showed proteinuria, hematuria or leukocyteuria. All patients were asymptomatic. Biopsy performed showed proliferative glomerular changes (mild to severe).

In our study 4 cases $(6.90 \%)$ of MPGN were seen. Two cases $(3.45 \%)$ were idiopathic, 1 case was positive for antinuclear antibody (SLE) and other positive for Mantoux test. All the cases were seen in females above the age of 8 years.Histology revealed increase in the measangial cellularity and matrix on H\&E staining, confirmed on periodic acid Schiff (PAS) stain . Silver stain showed splitting of the glomerular capillary basement membrane typically described as tram tracking (fig.6). Immunofluorescence study revealed strongly positive coarse, linear C3 deposits along the capillary wall. The 2 cases where immunofluorescence was available also showed IgG deposits in the mesangium, but were weakly positive. Electron microscopy revealed thickened capillary wall due to interposed mesangial cells, mesangial matrix and electron dense subendothelial deposits (fig.6D). In the study by Gulati et al. membranoproliferative glomerulonephritis was found in 2 cases. Both the cases were seen in males and presented with hematuria.

In our study light microscopy and immunopathology findings correlated with electron microscopy findings, in 20 cases out of the 24 cases $(80.95 \%)$ where electron microscopic diagnosis was available. Out of the 4 cases where diagnosis did not match, the impression on light microscopy was FSGS, which was interpreted as minimal change disease (2 cases) and diffuse mesangial proliferation (1 case) on electron microscopy. One case diagnosed as IgA nephropathy failed to show mesangial deposits on electron microscopy. Although classification of glomerular diseases based on ultrastructural findings has been attempted, these systems are less successful than histologic and / or immunopathologic schemes because of the limited sample studied by electron microscopy. ${ }^{[18]}$

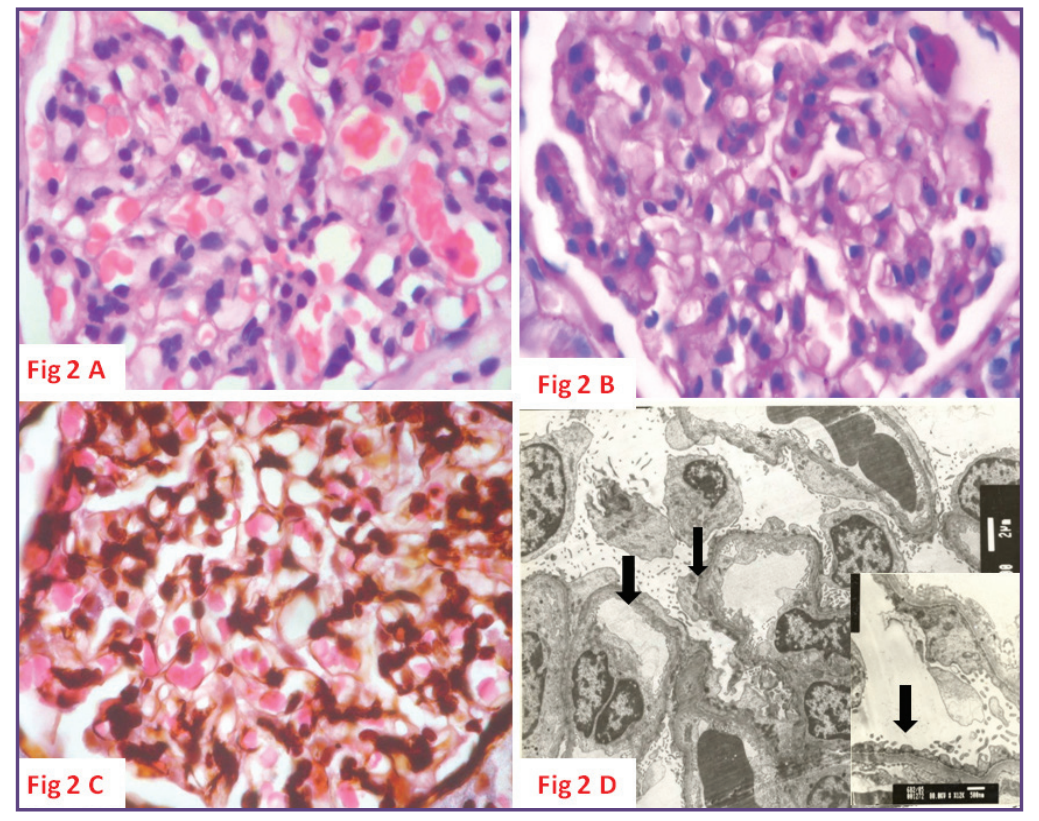

Fig. 2: Minimal Change Disaease (MCD): Unremarkable glomerulus with patent capillary lumina \& normal mesangial cellularity (H \&EX100)(2A). PAS stained section showing normal glomerular capillary wall thickness \& mesangium (X100) (2B). Absence of sclerosis, and normal glomerular capillary walls ( Silver, X100)(2C). Ultrastructure :EM- Note the flattening $\&$ fusion of the foot processes (arrows) of the visceral epithelial cells ( uranyl acetate lead citrate, X2,500)(2D). 

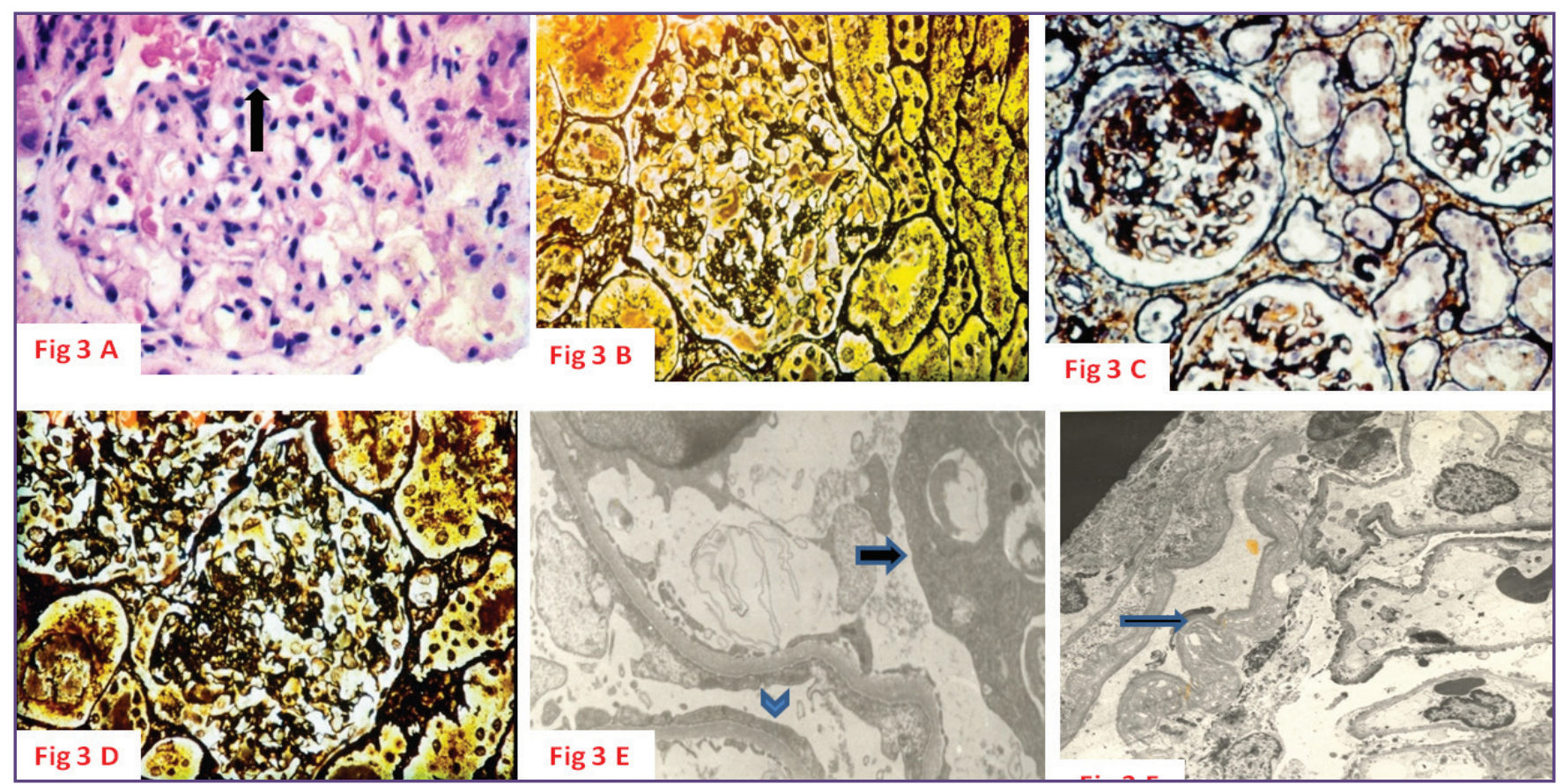

Fig. 3: FSGS. Focal sclerosis of the glomerulus with collapse of the capillary tuft(arrow) while rest of the glomerulus is unremarkable ( H\&E, X100) (3A) Silver stain highlighting the sclerosed area in the glomerulus(peripheral sclerosis)(3B), perihilar region of the glomerulus (3C), focal sclerosis of the glomerulus at the origin of the proximal convoluted tubule (tip lesion)(3D). [SilverX100]EM- focal area of sclerosis (arrow) and the flattening of the foot processes (arrow head). (3E)[uranyl lead citrate X8,000] \& the wrinkling of the glomerular basement membrane (arrow) (uranyl acetate lead citrate, X2,500)(3F).

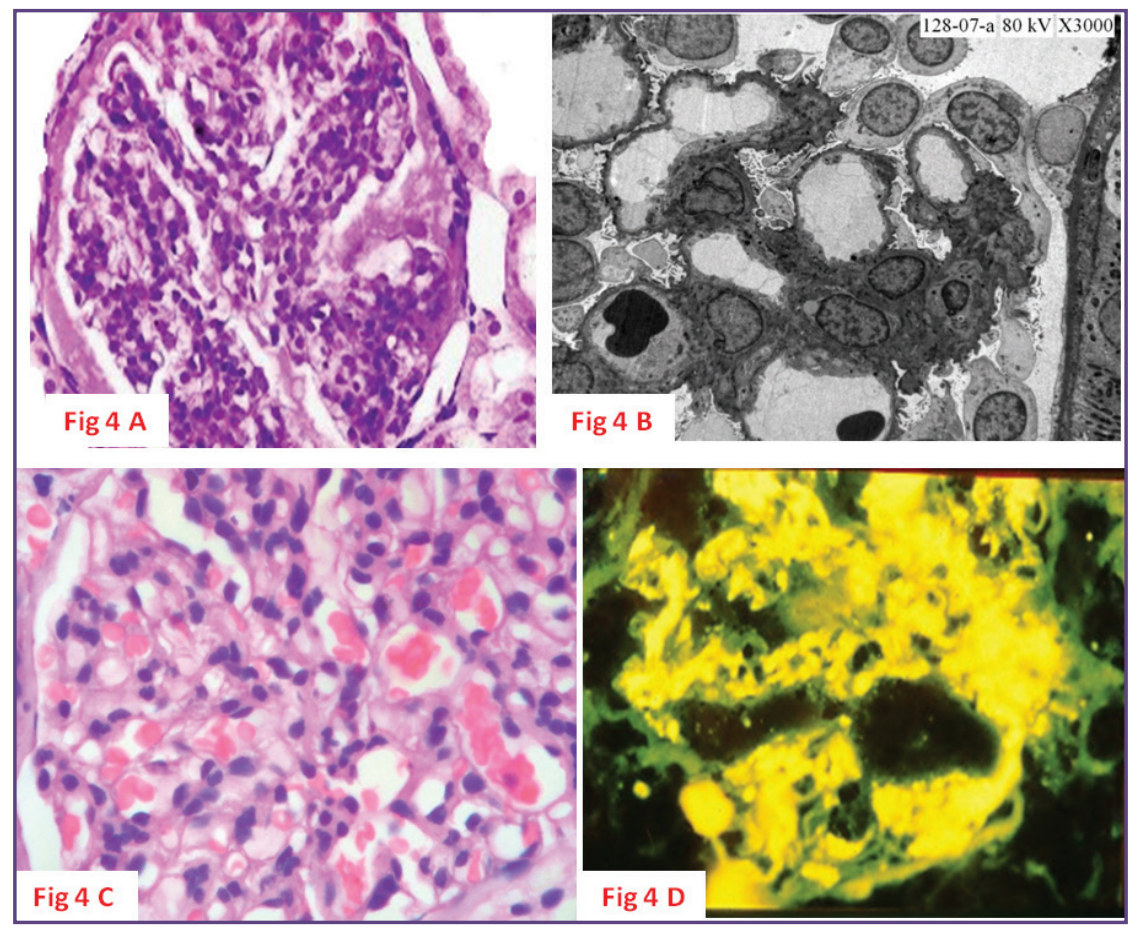

Fig. 4: MesPGN:Mesangium is expanded by increase in cellularity and matrix. (PAS100X)(4A)EM: electron-dense granular deposits within the mesangial matrix (4B) seen. IgM nephropathy: Unremarkable glomerulus (H \&EX100)(4C), Immunoflurescence showing bright and predominantly mesangial dense deposits.(Anti-IgM, X100)(4D). 


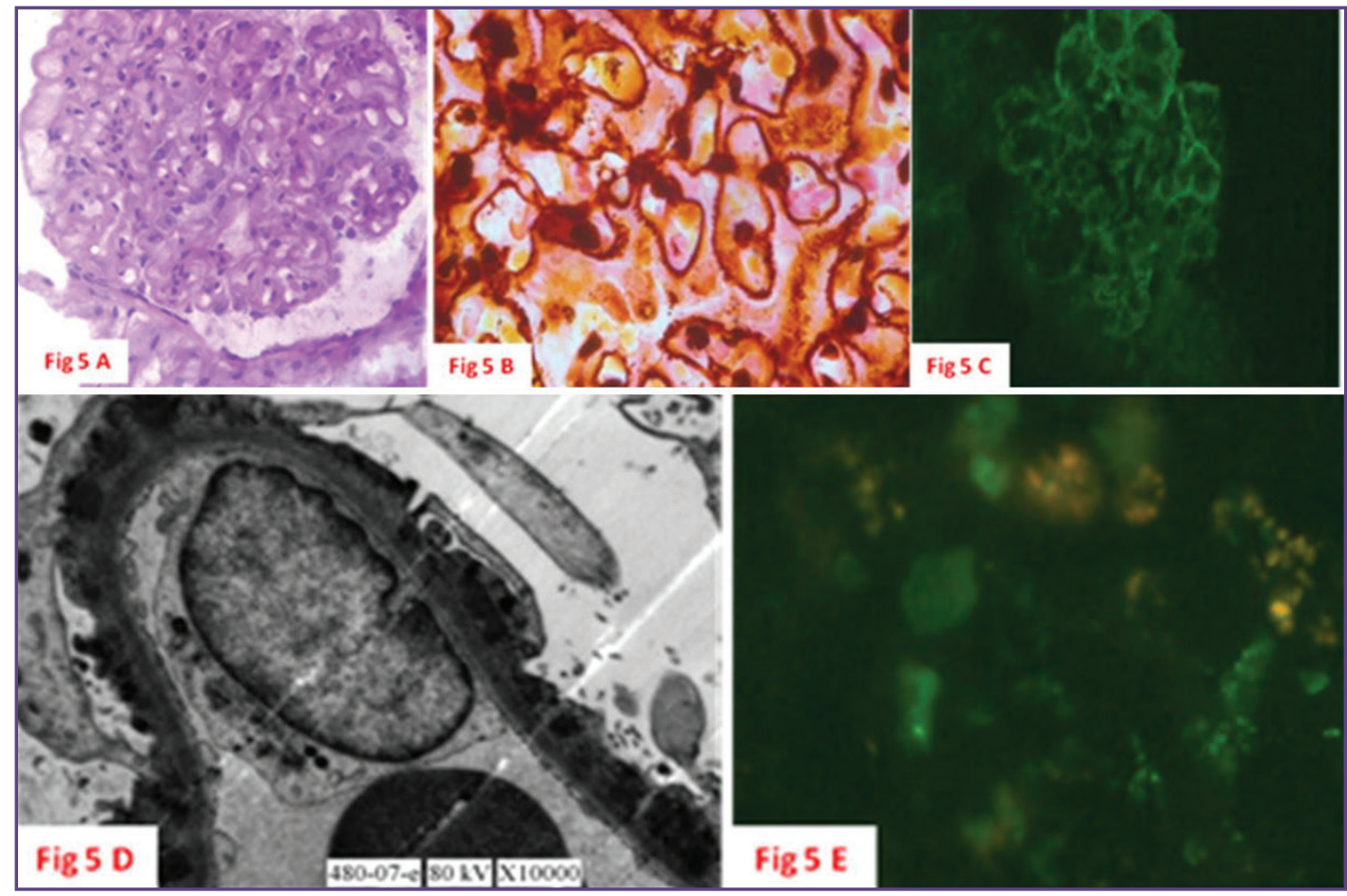

Fig. 5: Membranous GN: . Showing the thickening of the glomerular capillary walls without increase in the cellularity (PAS, X100)(5A) . Methanamine silver stain showing spikes on the subepithelial surface of glomerular basement membrane (X200)(5B). IF showing diffuse finely granular deposits outlining the glomerular capillary walls (Anti-IgG, X100)(5C).EM: sub epithelial deposits in contact with $\&$ indenting the visceral epithelial cells $(\mathrm{X15}, 000)(5 \mathrm{D})$. IgA nephropathy: Diffuse mesangial granular, lumpy Immunoflurescence with an anti-IgA antibody (Anti-IgA; X100)(5E).

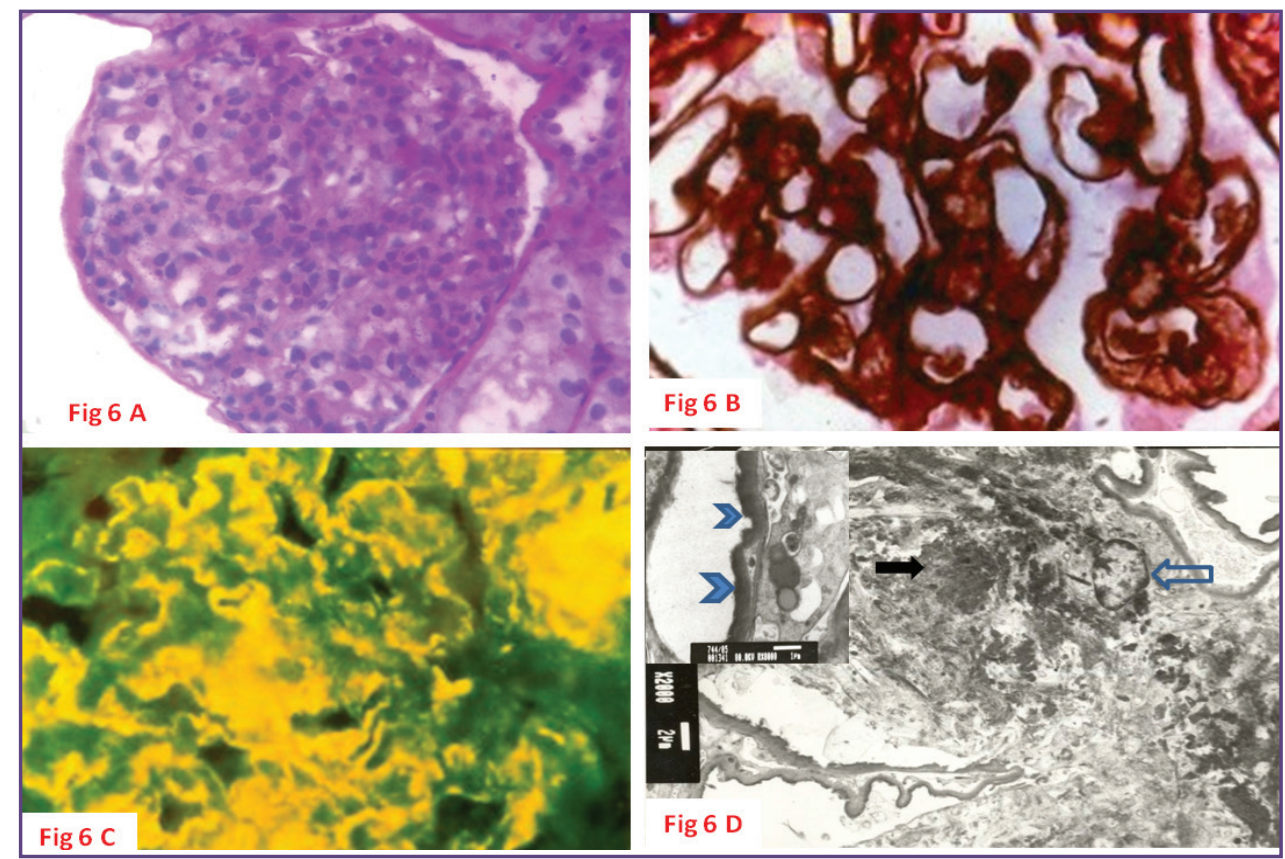

Fig 6 :Membranoproliferative GN: Glomerulus showing increase in the mesangial cellularity, lobular accentuation \& increase in mesangial matrix (H\&E, X45)(6A). Showing splitting (tram tracking) of the capillary basement membrane (Silver, X100)(6B). Immunoflurescence of MPGN type I. Note the diffuse, broad capillary loop \& mesangial deposits (Anti-C3, X100)(6C).EM: glomerular capillary wall due to mesangial cell interposition (open arrow). Note the increase in mesangial matrix \& the electron dense deposits.(black arrow) [uranyl acetate, lead citrate, X2000]Inset showing subendothelial deposits(arrow heads).(X8000)(6D) 


\section{Conclusion}

The country of origin may affect the proportions of the diseases with infections as etiology causing the nephrotic syndrome in paediatric age group, as in India. MCD still remains most common histomorphological lesion in paediatric nephrotic syndrome but rise in FSGS cases as a cause for nephrotic syndrome is now being encountered.

\section{Acknowledgements}

Dr.Arun Chitale, Senior Pathologist, Jaslok Hospital, Mumbai for Electron microscopy study

\section{Reference}

1. Abramowicz M, Barnett HL, Edelmann Jr. CM, et al. Controlled trial of Azithioprine in children with nephrotic syndrome: A report for International Kidney Diseases in Children. Lancet.1970; (i) 7654: 959-961.

2. 2. Sanjeev G, Debashish S, Sharma RK, et al. Steroid resistant nephrotic syndrome: role of histopathology. India Paediatrics.2006; 43:55- 60.

3. Mallick NP. Epidemiology and natural course of idiopathic nephrotic syndrome. Clin Nephrol.1991; 35: S3-S7.

4. Souilmi FZ, Houssaini TS, Alaoui H, et al. Indications and results of renal biopsy in children: A single-center experience from Morocco. Saudi J Kidney Dis Transpl. 2015; Jul-Aug;26(4):810-15.

5. Kumar J, Gulati S, Sharma AP, Sharma RK, Gupta RK. Histopathologic spectrum of childhood nephrotic syndrome in Indian children. Pediatr Nephrol.2003; 18:657-660.

6. Habib R, Kleinknecht C. The primary nephrotic syndrome in childhood: classification and clinicopathologic study of 406 cases. Sommers SC, ed. Pathology annual. 1971;417-474

7. White RHR, Glasgow EF, Mill RJ. Clinicopathological study of nephrotic syndrome in childhood. The Lancet.1970; i(7661):1353-1359.
8. Cho BS, Yoon SR, Jang JY, Pyun KH, Lee CH. Up-regulation of interleukin-4 and CD23/FcepsilonRII in minimal change nephrotic syndrome. Pediatr Nephrol.1999; 13: 199-204.

9. Yap HK, Cheung W, Murugasu B, Sim SK, Seah CC, Jordan SC.Th1 and Th2 cytokine mRNA profiles in childhood nephrotic syndrome: Evidence of increased IL-13 mRNA expression in relapse. J Am Soc Nephrol.1999; 10: 529-537.

10. Van Der Berg JG, aten J, Annink C, Ravesloot JH, Weber E, Weening JJ. IL-4 and IL-13 promote basolateral secretion of $\mathrm{H}(+)$ and cathepsin L by glomerular epithelial cells. Am J Physiol Renal Physiol.2002; 282: F26-F33.

11. Southwest Pediatric Nephrology Study Group. Childhood nephrotic syndrome

12. associated with diffuse mesangial hypercellularity. Kidney Int1983; 24: 87-94

13. Bonilla-Felix M, Parra C, Ferris M, et al. Changing patterns in the histopathology of idiopathic nephrotic syndrome. Kidney Int.1999; 55:1885-1890.

14. Waldherr R, Gubler MC, Levy M, Broyer M, Habib R. The significance of pure mesangial proliferation in idiopathic nephrotic syndrome. Clin Nephrol.1978; 10:171-179.

15. Bernstein J Jr, Edelmann CM Jr. Minimal change nephrotic syndrome: histopathology and steroid responsiveness. Arch Dis Child.1982; 57: 816.

16. Hirszel P, Yamase HT, Carney WR, et al. Mesangial proliferative glomerulonephritis with $\operatorname{IgM}$ deposists: clinicopathologic analysis and evidence for morphologic transitions. Nephron.1984; 38:100-108.

17. Berger J, Hinglais N. Intercapillary deposits of IgA-IgG. J Urol Nephrol (Paris). 1968;74(9):694-5

18. Sagel I, Treser G, Ty A, et al. Occurrence and nature of glomerular lesions after group A streptococci infections in children. Ann Intern Med. 1973;79:492-499.

19. Jennette JC, Olson MD, Silva MD. Heptinstall's Pathology of the Kidney. 7th ed. Philadelphia: Wolters Kluwer; 2014.

*Corresponding author:

Dr. Chetan Sudhakar Chaudhari, 11, Jagannath darshan, M.D.Keni Road, Near RBI Quarters, Bhandup Village (E), Mumbai, India

Phone: +91 9819133606

Email: drchetanchaudhari26@yahoo.co.in

Financial or other Competing Interests: None.

Date of Submission : 31.07.2016

Date of Acceptance : 23.11.2016

Date of Publication : 04.02.2017 